\title{
Fatores associados ao contato pele a pele entre mãe/filho e amamentação na sala de parto
}

\author{
Factors associated with skin to skin \\ contact between mother/son and \\ breastfeeding in the delivery room
}

Cristianny Miranda e SILVA ${ }^{1}$

Simone Cardoso Lisboa PEREIRA ${ }^{2}$

leda Ribeiro PASSOS 3

Luana Caroline dos SANTOS 2

\section{R E S U M O}

\section{Objetivo}

Investigar fatores associados ao contato pele a pele entre mãe e filho e à amamentação na sala de parto entre nutrizes atendidas em um banco de leite humano.

\section{Métodos}

Foram avaliadas 12283 mães em estudo retrospectivo (2009-2012) com dados secundários obtidos de protocolo estruturado. Adotou-se modelo de Poisson com abordagem hierarquizada em níveis distal, intermediário e proximal para dados sociodemográficos maternos; pré-natais e gestacionais; do bebê; e atenção hospitalar.

\section{Resultados}

Estiveram associados, respectivamente, à maior prevalência do contato pele a pele e da amamentação na sala de parto: parto normal ( $R P=1,34$; IC95\% 1,27-1,41 e RP=1,63; IC95\% 1,45-1,83) e sem complicação ( $R P=1,24$;

\footnotetext{
1 Universidade Federal de Minas Gerais, Faculdade de Medicina, Programa de Pós-Graduação em Ciências da Saúde - Saúde da Criança e do Adolescente. Av. Professor Alfredo Balena, 190, Sala 324, 30130-100, Belo Horizonte, MG, Brasil. Correspondência para/Correspondence to: CM SILVA. E-mail: <cristiannyms@gmail.com>.

2 Universidade Federal de Minas Gerais, Escola de Enfermagem, Departamento de Nutrição. Belo Horizonte, MG, Brasil.

3 Enfermeira. Belo Horizonte, MG, Brasil.

Apoio: Fundação de Amparo à Pesquisa do Estado de Minas Gerais (Processo no APQ-01782-10).

Artigo elaborado a partir da dissertação de CM SILVA, intitulada: "Avaliação do incentivo ao aleitamento materno e seus fatores associados em um banco de leite humano referência em Minas Gerais”, Universidade Federal de Minas Gerais; 2016.
} 
IC95\% 1,12-1,38 e RP=1,27; IC95\% 1,03-1,56), peso ao nascer adequado (RP=1,23; IC95\% 1,11-1,36 e $R P=1,92$; IC95\% 1,48-2,48) e a termo ( $R P=1,18$; IC95\% 1,10-1,28 e RP=1,40; IC95\% 1,17-1,67).

\section{Conclusão}

A forte influência da atenção hospitalar e as condições ao nascer do recém-nascido explicaram os desfechos em estudo. Ressalta-se a necessidade da adoção de medidas que priorizem o contato pele a pele e a amamentação na sala de parto nos grandes hospitais da capital, como a redução ou adiamento de intervenções na assistência pós-parto. Estudos longitudinais poderão esclarecer outras questões sobre o tema.

Palavras-chave: Aleitamento materno. Bancos de leite. Período pós-parto. Relações mãe-filho.

\section{A B S T R A C T}

\section{Objective}

To investigate factors associated with skin to skin contact between mother and child and breastfeeding at the delivery room in mothers who visit a human milk bank.

\section{Methods}

This retrospective study (2009 to 2012) assessed the secondary data of 12,283 mothers obtained by a structured protocol. A distal-, intermediate-, and proximal-level hierarchical Poisson regression model analyzed the sociodemographic, maternal, prenatal, gestational, infant, and hospital care data.

\section{Results}

The following items were associated with a higher prevalence of skin to skin contact and breastfeeding in the delivery room: vaginal delivery $(R P=1.34 ; 95 \% C l$ 1.27-1.41 and $P R=1.63 ; 95 \% C l$ 1.45-1.83), uneventful delivery $(P R=1.24 ; 95 \% C l 1.12-1.38$ and $P R=1.27 ; 95 \% C l$ 1.03-1.56), appropriate birth weight $(P R=1.23 ; 95 \% C l 1.11-1.36$ and $P R=1.92 ; 95 \% \mathrm{Cl} 1.48-2.48)$, and term birth ( $P R=1.18 ; 95 \% \mathrm{Cl} 1.10-1.28$ and $P R=1.40 ; 95 \% \mathrm{Cl} 1.17-1.67)$.

\section{Conclusion}

The strong influence of hospital care and newborn birth conditions explained the study outcomes. Actions that promote skin to skin contact and breastfeeding in the delivery room of large hospitals in capital cities are needed, such as reduction or postponement of interventions during postnatal care. Longitudinal studies may clarify other issues on this subject.

Keywords: Breast feeding. Milk banks. Postpartum period. Mother-child relations.

\section{N T R O D U Ç Ã O}

O contato pele a pele precoce entre mãe e filho e a amamentação na primeira hora de vida do bebê representam importantes estratégias para a promoção do Aleitamento Materno (AM) ${ }^{1}$. Essas orientações são preconizadas pela Organização Mundial da Saúde (OMS) e pelo Fundo das Nações Unidas para a Infância (UNICEF, United Nations Children's Found) e correspondem ao passo 4 dos Dez Passos para o Sucesso do Aleitamento Materno da Iniciativa Hospital Amigo da Criança (IHAC), os quais representam um conjunto de metas em prol da amamentação natural ${ }^{2}$.

O passo 4 - ajudar as mães a iniciar a amamentação na primeira meia hora após o nasci- mento ${ }^{3}$ - recomenda colocar o recém-nascido em contato pele a pele com a mãe no pós-parto imediato por, pelo menos, uma hora e ajudar a puérpera a perceber o momento em que a criança está pronta para mamar, oferecendo ajuda, caso necessário. Esse contato precoce significa colocar o bebê nu em posição prona sobre o peito da mãe com o intuito de favorecer a adaptação na transição do espaço intra para o extrauterino, incentivando a amamentação imediatamente após o parto ${ }^{4}$.

Essa estratégia visa a promoção, proteção e apoio ao AM e se baseia na capacidade de interação dos recém-nascidos com suas mães, logo após o nascimento. Assim, a amamentação deve ser incentivada ainda na sala de parto, desde 
que mãe e filho estejam bem e interagindo. $\mathrm{O}$ contato pele a pele precoce também é fundamental para o estabelecimento do vínculo mãe-filho, reduzindo o choro e o estresse do recém-nascido, mantendo-o aquecido pela transmissão do calor de sua mãe, além de estar relacionado com o aumento da duração da amamentação e do aleitamento materno exclusivo ${ }^{1,5,6}$.

Apesar da reconhecida importância, alguns hábitos e rotinas hospitalares podem dificultar esses processos, como: cuidados imediatos ao recém-nascido, maior incidência de cesarianas e consequente redução do estado de alerta do bebê e acentuada analgesia do parto, acarretando sonolência materna ${ }^{6}$. De modo semelhante, dificuldades têm sido apontadas quanto à amamentação na primeira hora de vida, relacionadas à resistência dos profissionais, ao desconhecimento das vantagens dessa prática e à política da instituição?

De acordo com a Organização Pan-Americana da Saúde ${ }^{8}$, somente em oito dos 14 países que dispõem de dados, $50 \%$ ou mais dos recém-nascidos são colocados para mamar no peito uma hora após o parto. No Brasil, a Pesquisa Nacional de Demografia e Saúde da Criança e da Mulher (PNDS), $2006^{9}$ demonstrou que as práticas do contato pele a pele e da amamentação na primeira hora de vida foram realizadas em apenas 42,9\% das crianças brasileiras. Já a II Pesquisa de Prevalência de Aleitamento Materno, realizada em 2008, denotou que, do total de crianças analisadas, apenas $67,7 \%$ mamaram na primeira hora de vida, variando de 58,5\% em Salvador (BA) a $83,5 \%$ em São Luís $(M A)^{10}$.

Ademais, em outro estudo nacional, de base hospitalar, notou-se que o contato pele a pele logo após o nascimento foi mais frequente na Região Sul (32,5\%), bem como a amamentação na sala de parto $(22,4 \%)$. Entretanto, as proporções observadas com relação à esta prática demonstraram ainda serem baixas em todas as regiões do Brasil (16,1\%), sendo que o Nordeste apresenta a menor delas $(11,5 \%)^{11}$.
Nesse cenário, e com o intuito de fortalecer as políticas públicas de saúde voltadas para o incentivo ao AM, os Bancos de Leite Humano (BLH) têm cumprido importante papel assistencial junto às puérperas e nutrizes, no sentido de promover, proteger e apoiar o AM. Para isso, acompanham as mulheres que apresentam dificuldades na prática do aleitamento, além de realizarem a coleta, processamento e controle de qualidade do colostro, leite de transição e leite maduro ${ }^{12}$. Assim, considerando a relevância do contato pele a pele e da amamentação na sala de parto como condições intrínsecas à política de AM no país e aos programas de incentivo à amamentação, o presente trabalho pretendeu investigar os fatores sociodemográficos e pessoais, relativos ao pré-natal, à gestação, à atenção hospitalar e ao recém-nascido que influenciam esses desfechos em nutrizes atendidas em um banco de leite humano.

\section{M É T O D O S}

Trata-se de um estudo retrospectivo com dados secundários, obtidos a partir de protocolo de atendimento padronizado, de nutrizes atendidas em um BLH de uma maternidade pública de uma metrópole brasileira. Em 1986, essa maternidade tornou-se referência em gestação de alto risco e, no ano de 1999, recebeu o título de Hospital Amigo da Criança, concedido pela OMS, UNICEF e Ministério da Saúde ${ }^{13}$.

O Banco de Leite Humano da referida maternidade é destinado, principalmente, às mães que demonstram dificuldade para amamentar ou que queiram doar o excedente de leite. O serviço também desenvolve ações de promoção, proteção e apoio à amamentação através de atividades assistenciais e educativas oferecidas às mulheres as quais apresentam intercorrências ou dificuldades na prática da amamentação, além da coleta, processamento, controle de qualidade e distribuição do leite humano pasteurizado ${ }^{14}$. As mulheres deste estudo procediam, em sua maioria, dessa mesma maternidade ou de outros hospitais 
públicos e privados, localizados na capital estadual ou no interior do estado.

Foram avaliadas as informações presentes no referido formulário de atendimento das mães que procuraram o serviço do BLH, encaminhadas pelo médico ou por iniciativa própria, no período de 2009 a 2012. Os desfechos - contato pele a pele precoce entre mãe e recém-nascido (sim/não) e AM na sala de parto (sim/não) - foram obtidos a partir desses protocolos de atendimento. Não foi realizado cálculo amostral, pois foram avaliadas todas as mulheres atendidas no local no período referido. Esse protocolo era preenchido manualmente pela equipe de saúde do BLH através de entrevista com as nutrizes no momento em que eram recepcionadas para o atendimento de intercorrências ou aconselhamento em AM.

Elegeu-se, para a análise, 21 variáveis do formulário, as quais se relacionavam aos desfechos de interesse: características sociodemográficas e pessoais maternas (idade em anos: <20; 20 a 29; 30 a 39; 40 ou mais; local de residência; escolaridade; profissão; estado civil: solteira, casada, outros; morbidade referida: sim/não; número de filhos: 0, 1, 2, 3 ou mais; e amamentação anterior: sim/não), características da assistência ao pré-natal (realização do pré-natal: sim/não; local de realização do pré-natal: setor público, privado ou não realizado; e número de consultas pré-natais realizadas ${ }^{15}$ : até 3, 4 a 6, acima de 6; recebimento de orientações sobre AM no pré-natal: sim/não; e tipo de orientação sobre AM recebida nesse período: individual ou em grupo), informações gestacionais (uso de medicamentos: sim/não; tabagismo durante a gravidez: sim/não; e presença de gestação múltipla: sim/não), características da assistência hospitalar (tipo de hospital; tipo de parto: normal, cesárea ou fórceps; e presença de complicações no parto: sim/não) e informações a respeito do recém-nascido (peso ao nascer ${ }^{15}$. baixo peso $(<2500 \mathrm{~g})$ ou peso adequado ( $\geq 2500 \mathrm{~g})$; e idade gestacional ao nascimento: pré-termo ${ }^{15}$ (<37 semanas) ou a termo (38 a 42 semanas)).
O local de residência das mães compreendeu: a capital do estado, Belo Horizonte; a região metropolitana, a qual engloba um conjunto de municípios próximos entre si e que são integrados socioeconomicamente à capital estadual; e as demais cidades do interior do estado. Quanto à profissão, a categoria referente às profissões remuneradas se referiu ao trabalho formal e informal (respectivamente, com ou sem carteira assinada), desde que as mulheres recebessem remuneração.

Já a variável tipo de hospital se refere ao local de realização do parto mencionado pelas mães durante o preenchimento do formulário de atendimento. A categoria HAC se refere à maternidade sede do $B L H$, onde foi realizada a presente pesquisa. Os grandes hospitais da capital representam quatro hospitais particulares situados na capital do estado e que foram bastante citados pelas mulheres. A categoria outros diz respeito aos outros hospitais, públicos e privados, situados na capital ou no interior do estado, relatados pelas participantes durante o preenchimento do protocolo.

Com relação à orientação referente à amamentação, a individual diz respeito à orientação oferecida pelo médico ou equipe de saúde multidisciplinar durante as consultas pré-natais por meio de contato pessoal, face a face, com as gestantes. Já a orientação em grupo se refere à vivência coletiva com outras mulheres e profissionais de saúde treinados acerca da temática durante o período pré-natal.

Os dados obtidos foram analisados, após avaliação de consistência, com o auxílio dos programas Statistical Package for the Social Sciences ${ }^{\circledR}$ (SPSS) versão 19.0 e Stata versão 11.2. Realizou-se análise descritiva com cálculo de frequências absolutas e relativas, medidas de tendência central e dispersão. Ademais, foram realizados os testes Kolmogorov-Smirnov, para verificar a adesão das variáveis quantitativas à distribuição normal, teste Qui-quadrado, para comparação de proporções, e regressão de Poisson. Adotou-se nível de significância de $5 \%(p<0,05)$ para todas as análises. 
Análise bivariada (teste Qui-quadrado de Pearson) foi realizada para cada variável, a fim de verificar a associação com os desfechos. Aquelas com valor de $p<0,20$ foram hierarquizadas por nível de proximidade com os desfechos e em grupos, a partir de revisão da literatura': distais (características sociodemográficas e pessoais maternas - modelo 1), intermediárias (assistência ao pré-natal e características gestacionais maternas - modelo 2) e proximais (características da assistência hospitalar e do recém-nascido - modelo 3) (Figura 1).

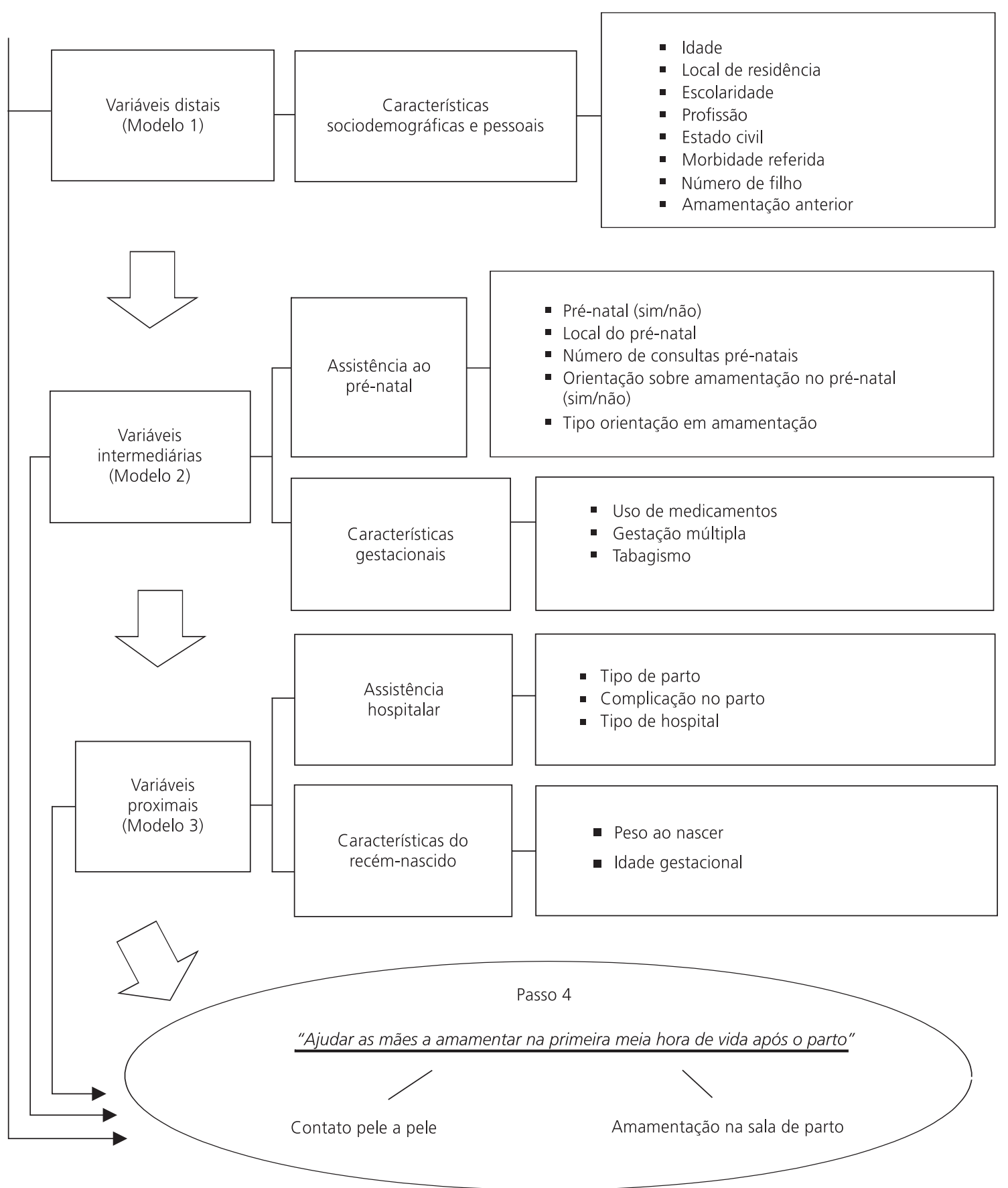

Figura 1. Contato pele a pele e amamentação na sala de parto, segundo modelo hierarquizado. Banco de Leite Humano, Belo Horizonte (MG), 2009-2012. 
Em seguida, essas variáveis foram selecionadas para análise multinível de Poisson com variância robusta e hierarquizada e eliminação Backward. Isso possibilitou avaliar o efeito dos grupos de variáveis em relação à proximidade com os desfechos. Para isso, aquelas que apresentaram significância estatística em relação ao modelo 1 foram analisadas em conjunto com as do modelo 2 e, as resultantes dessa análise, com as variáveis do modelo 3, obtendo-se, assim, o modelo final ajustado por meio do Teste Hosmer e Lemeshow. No que se refere aos aspectos éticos, ressalta-se que esse estudo faz parte do projeto Banco de Leite Humano de Referência em Minas Gerais: caracterização, intervenção e avaliação, aprovado pelos Comitês de Ética envolvidos (Parecer $n^{\circ}$ 042/2010 e n ETIC 0079.0.203.000-10).

\section{RESULTADOS}

Foram avaliadas 12283 mães no período analisado, com mediana de idade de 29 (12 - 54) anos: 2225 (18,1\%) em 2009; 4279 (34,8\%) em 2010; 2970 (24,2\%) em 2011; e 2809 $(22,9 \%)$ em 2012. A maioria das mulheres teve seu parto realizado em hospitais do setor público (aproximadamente $64,0 \%$ ) e eram oriundas do Hospital Amigo da Criança, sede do BLH, analisado na presente pesquisa $(54,9 \%)$.

Os dados sociodemográficos e pessoais, de assistência ao pré-natal e hospitalares, gestacionais e do recém-nascido estão apresentados na Tabela 1. Na análise univariada, das 21 variáveis selecionadas para este estudo, "estado civil" não se associou ao desfecho "contato pele a pele" $(p>0,05)$. As variáveis "realização do pré-natal" e "uso de medicamentos" não se associaram à variável principal "amamentação na sala de parto"; além disso, "tabagismo" não apresentou associação com ambos os desfechos $(p>0,05)$ (Tabela 2). Destas, apenas "uso de medicamentos" foi recrutada para análise multivariada $(p<0,20)$.

Tabela 1. Características sociodemográficas e pessoais, da assistência ao pré-natal e hospitalar, gestacionais e do recém-nascido da amostra estudada. Banco de Leite Humano, Belo Horizonte (MG), 2009-2012.

\begin{tabular}{|c|c|c|c|c|}
\hline Características da amostra & Variáveis & Categoria & $n$ & $\%$ \\
\hline \multirow[t]{20}{*}{ Sociodemográficas e pessoais } & \multirow[t]{4}{*}{ Idade (anos) } & $<20$ & 1522 & 13,0 \\
\hline & & 20 a 29 & 4780 & 41,0 \\
\hline & & 30 a 39 & 4916 & 42,1 \\
\hline & & 40 ou mais & 450 & 3,9 \\
\hline & \multirow[t]{3}{*}{ Local de residência } & Belo Horizonte & 7161 & 71,6 \\
\hline & & Região metropolitana & 2374 & 23,8 \\
\hline & & Demais cidades & 460 & 4,6 \\
\hline & \multirow[t]{5}{*}{ Escolaridade } & Analfabeto & 31 & 0,3 \\
\hline & & Ensino fundamental & 2559 & 24,1 \\
\hline & & Ensino médio & 4325 & 40,7 \\
\hline & & Ensino técnico & 166 & 1,6 \\
\hline & & Graduação ou mais & 3541 & 33,3 \\
\hline & \multirow[t]{3}{*}{ Profissão } & Do lar & 1927 & 19,8 \\
\hline & & Estudante & 524 & 5,4 \\
\hline & & Profissões remuneradas & 7257 & 74,8 \\
\hline & \multirow[t]{3}{*}{ Estado civil } & Solteira & 4123 & 38,0 \\
\hline & & Casada & 6595 & 60,8 \\
\hline & & Outros & 131 & 1,2 \\
\hline & \multirow[t]{2}{*}{ Morbidade referida } & Sim & 1967 & 20,6 \\
\hline & & Não & 7573 & 79,4 \\
\hline
\end{tabular}


Tabela 1. Características sociodemográficas e pessoais, da assistência ao pré-natal e hospitalar, gestacionais e do recém-nascido da amostra estudada. Banco de Leite Humano, Belo Horizonte (MG), 2009-2012.

\begin{tabular}{|c|c|c|c|c|}
\hline Características da amostra & Variáveis & Categoria & $\mathrm{n}$ & $\%$ \\
\hline & Número de filhos & 0 & 69 & 0,8 \\
\hline & & 1 & 5177 & 61,6 \\
\hline & & 2 & 1600 & 19,1 \\
\hline & & 3 ou mais & 1554 & 18,5 \\
\hline & Amamentação anterior & Sim & 2851 & 38,9 \\
\hline & & Não & 4478 & 61,1 \\
\hline \multicolumn{5}{|l|}{ Assistência ao pré-natal } \\
\hline & Realização e local do pré-natal & Sim, público & 6181 & 52,4 \\
\hline & & Sim, privado & 5468 & 46,3 \\
\hline & & Não realizado & 150 & 1,3 \\
\hline & $\mathrm{N}^{\circ}$ consultas pré-natais ${ }^{\mathrm{a}}$ & Até 3 & 285 & 2,6 \\
\hline & & De 4 a 6 & 1168 & 10,8 \\
\hline & & Acima de 6 & 9349 & 86,6 \\
\hline & Orientação sobre AM & Sim, individual & 2410 & 23,9 \\
\hline & & Sim, em grupo & 1496 & 14,9 \\
\hline & & Não & 6168 & 61,2 \\
\hline \multicolumn{5}{|l|}{ Gestacionais } \\
\hline & Uso de medicamentos & $\operatorname{Sim}$ & 2027 & 19,6 \\
\hline & & Não & 8327 & 80,4 \\
\hline & Tabagismo & $\operatorname{sim}$ & 569 & 6,2 \\
\hline & & Não & 8660 & 93,8 \\
\hline & Gestação múltipla & Sim & 85 & 0,9 \\
\hline & & Não & 9202 & 99,1 \\
\hline \multicolumn{5}{|l|}{ Assistência hospitalar } \\
\hline & Tipo de parto & Normal & 4905 & 41,6 \\
\hline & & Cesárea & 6574 & 55,7 \\
\hline & & Fórceps & 319 & 2,7 \\
\hline & Complicações no parto & $\operatorname{Sim}$ & 1138 & 12,1 \\
\hline & & Não & 8274 & 87,9 \\
\hline & Tipo de hospital & HAC & 6500 & 54,9 \\
\hline & & Grandes hospitais da capital & 3843 & 32,4 \\
\hline & & Outros & 1504 & 12,7 \\
\hline \multicolumn{5}{|l|}{ Recém-nascido } \\
\hline & Peso ao nascer & Baixo peso & 1532 & 16,5 \\
\hline & & Peso adequado & 7755 & 83,5 \\
\hline & Idade gestacional ao nascer & RN pré-termo & 2384 & 27,3 \\
\hline & & $\mathrm{RN}$ a termo & 6339 & 72,7 \\
\hline \multicolumn{5}{|l|}{ Variáveis desfecho } \\
\hline & Contato pele a pele & $\operatorname{Sim}$ & 6309 & 60,5 \\
\hline & & Não & 4111 & 39,5 \\
\hline & Amamentação na sala de parto & Sim & 2267 & 25,5 \\
\hline & & Não & 6625 & 74,5 \\
\hline
\end{tabular}

Nota: aFonte: Brasil (2005) ${ }^{15}$

AM: Aleitamento Materno; HAC: Hospital Amigo da Criança; RN: Recém-nascido

Também foi observada maior frequência procedimento foi realizado no Hospital Amigo da de complicações no parto entre as mulheres cujo Criança da presente pesquisa $(15,1 \%)$ em compa- 
Tabela 2. Fatores associados ao contato pele a pele e à amamentação na sala de parto. Banco de Leite Humano, Belo Horizonte (MG), 2009-2012.

1 de 2

\begin{tabular}{|c|c|c|c|c|c|c|c|}
\hline \multirow{3}{*}{ Variáveis } & \multirow{3}{*}{ Categorias } & \multicolumn{6}{|c|}{ Passo 4} \\
\hline & & \multicolumn{3}{|c|}{ Contato pele a pele } & \multicolumn{3}{|c|}{ AM na sala de parto } \\
\hline & & $\operatorname{Sim}(\%)$ & Não (\%) & Valor $p^{\text {a }}$ & $\operatorname{Sim}(\%)$ & Não (\%) & Valor $p^{\text {a }}$ \\
\hline \multicolumn{8}{|l|}{ Idade } \\
\hline & $<20$ & 63,9 & 36,1 & $<0,001$ & 27,8 & 72,2 & 0,01 \\
\hline & 20 a 29 & 62,5 & 37,5 & & 26,8 & 73,2 & \\
\hline & 30 a 39 & 59,9 & 40,1 & & 24,3 & 75,7 & \\
\hline & 40 ou mais & 55,5 & 44,5 & & 21,3 & 78,7 & \\
\hline \multicolumn{8}{|c|}{ Local de residência } \\
\hline & Belo Horizonte & 58,8 & 41,2 & $<0,001$ & 25,6 & 74,4 & $<0,001$ \\
\hline & Região metropolitana & 59,3 & 40,7 & & 26,7 & 73,3 & \\
\hline & Demais cidades & 41,0 & 59,0 & & 13,8 & 86,2 & \\
\hline \multicolumn{8}{|c|}{ Escolaridade } \\
\hline & Analfabeto & 74,1 & 25,9 & 0,01 & 29,6 & 70,4 & $<0,001$ \\
\hline & Ensino fundamental & 60,9 & 39,1 & & 28,6 & 71,4 & \\
\hline & Ensino médio & 60,1 & 39,9 & & 27,5 & 72,5 & \\
\hline & Ensino técnico & 58,8 & 41,2 & & 25,2 & 74,8 & \\
\hline & Graduação ou mais & 57,0 & 43,0 & & 22,5 & 77,5 & \\
\hline \multicolumn{8}{|l|}{ Profissão } \\
\hline & Do lar & 62,6 & 37,4 & 0,02 & 28,4 & 71,6 & 0,01 \\
\hline & Estudante & 60,1 & 39,9 & & 25,9 & 74,1 & \\
\hline & Remunerada & 58,7 & 41,3 & & 24,4 & 75,6 & \\
\hline \multicolumn{8}{|c|}{ Estado civil } \\
\hline & Solteira & 61,1 & 38,9 & 0,32 & 28,0 & 72,0 & $<0,001$ \\
\hline & Casada & 59,6 & 40,4 & & 24,3 & 75,7 & \\
\hline & Outros & 61,1 & 38,9 & & 24,7 & 75,3 & \\
\hline \multicolumn{8}{|c|}{ Morbidade referida } \\
\hline & Sim & 55,6 & 44,4 & $<0,001$ & 22,3 & 77,7 & $<0,001$ \\
\hline & Não & 60,1 & 39,9 & & 28,3 & 71,7 & \\
\hline \multicolumn{8}{|c|}{ Número de filhos } \\
\hline & 0 & 67,3 & 32,7 & 0,06 & 20,0 & 80,0 & $<0,001$ \\
\hline & 1 & 59,2 & 40,8 & & 21,0 & 79,0 & \\
\hline & 2 & 61,8 & 38,2 & & 27,1 & 72,9 & \\
\hline & 3 ou mais & 62,7 & 37,3 & & 24,1 & 75,9 & \\
\hline \multicolumn{8}{|c|}{ Amamentação anterior } \\
\hline & Sim & 61,9 & 38,1 & $<0,001$ & 29,8 & 70,2 & $<0,001$ \\
\hline & Não & 57,7 & 42,3 & & 24,1 & 75,9 & \\
\hline \multicolumn{8}{|l|}{ Pré-natal } \\
\hline & Sim & 60,9 & 39,1 & 0,01 & 25,7 & 74,3 & 0,33 \\
\hline & Não & 48,5 & 51,5 & & 20,7 & 79,3 & \\
\hline \multicolumn{8}{|c|}{ Local pré-natal } \\
\hline & Sim, público & 62,8 & 37,2 & $<0,001$ & 28,6 & 71,4 & $<0,001$ \\
\hline & Sim, privado & 58,9 & 41,1 & & 22,6 & 77,4 & \\
\hline & Não realizado & 48,5 & 51,5 & & 20,7 & 79,3 & \\
\hline \multicolumn{8}{|c|}{$\mathrm{N}^{\circ}$ consultas pré-natais ${ }^{\mathbf{b}}$} \\
\hline & Até 3 & 43,8 & 56,2 & $<0,001$ & 16,0 & 84,0 & $<0,001$ \\
\hline & De 4 a 6 & 61,4 & 38,6 & & 22,1 & 77,9 & \\
\hline & Acima de 6 & 62,3 & 37,7 & & 26,2 & 73,8 & \\
\hline
\end{tabular}


Tabela 2. Fatores associados ao contato pele a pele e à amamentação na sala de parto. Banco de Leite Humano, Belo Horizonte (MG), 2009-2012.

\begin{tabular}{|c|c|c|c|c|c|c|c|}
\hline \multirow{3}{*}{ Variáveis } & \multirow{3}{*}{ Categorias } & \multicolumn{6}{|c|}{ Passo 4} \\
\hline & & \multicolumn{3}{|c|}{ Contato pele a pele } & \multicolumn{3}{|c|}{ AM na sala de parto } \\
\hline & & $\operatorname{sim}(\%)$ & Não (\%) & Valor $p^{\mathrm{a}}$ & $\operatorname{Sim}(\%)$ & Não (\%) & Valor $p^{\mathbf{a}}$ \\
\hline \multicolumn{8}{|c|}{ Orientação sobre AM } \\
\hline & $\operatorname{sim}$ & 64,9 & 35,1 & $<0,001$ & 27,9 & 72,1 & $<0,001$ \\
\hline & Não & 59,8 & 40,2 & & 23,3 & 76,7 & \\
\hline \multicolumn{8}{|c|}{ Tipo orientação sobre AM } \\
\hline & Sim, individual & 64,9 & 35,1 & $<0,001$ & 29,6 & 70,4 & $<0,001$ \\
\hline & Sim, em grupo & 64,9 & 35,1 & & 25,2 & 74,8 & \\
\hline & Não & 59,8 & 40,2 & & 23,3 & 76,7 & \\
\hline \multicolumn{8}{|c|}{ Uso de medicamentos } \\
\hline & $\operatorname{sim}$ & 57,1 & 42,9 & $<0,001$ & 23,5 & 76,5 & 0,18 \\
\hline & Não & 60,8 & 39,2 & & 25,2 & 74,8 & \\
\hline \multicolumn{8}{|c|}{ Tabagismo } \\
\hline & $\operatorname{sim}$ & 60,3 & 39,7 & 0,27 & 23,2 & 76,8 & 0,36 \\
\hline & Não & 57,8 & 42,2 & & 25,1 & 74,9 & \\
\hline \multicolumn{8}{|c|}{ Gestação múltipla } \\
\hline & $\operatorname{sim}$ & 40,3 & 59,7 & $<0,001$ & 11,3 & 88,7 & 0,01 \\
\hline & Não & 58,7 & 41,3 & & 25,9 & 74,1 & \\
\hline \multicolumn{8}{|c|}{ Tipo de parto } \\
\hline & Normal & 71,6 & 28,4 & $<0,001$ & 34,4 & 65,6 & $<0,001$ \\
\hline & Cesárea & 51,8 & 48,2 & & 18,7 & 81,3 & \\
\hline & Fórceps & 67,9 & 32,1 & & 30,2 & 69,8 & \\
\hline \multicolumn{8}{|c|}{ Complicação no parto } \\
\hline & $\operatorname{sim}$ & 44,4 & 55,6 & $<0,001$ & 18,5 & 81,5 & $<0,001$ \\
\hline & Não & 63,1 & 36,9 & & 25,9 & 74,1 & \\
\hline & HAC & 63,2 & 36,8 & $<0,001$ & 28,1 & 71,9 & $<0,001$ \\
\hline & Grandes hospitais da capital & 55,0 & 45,0 & & 15,9 & 84,1 & \\
\hline & Outros & 65,0 & 35,0 & & 40,8 & 59,2 & \\
\hline \multicolumn{8}{|c|}{ Peso ao nascer } \\
\hline & Baixo peso & 40,6 & 59,4 & $<0,001$ & 12,9 & 87,1 & $<0,001$ \\
\hline & Peso adequado & 61,6 & 38,4 & & 28,0 & 72,0 & \\
\hline & RN pré-termo & 45,7 & 54,3 & $<0,001$ & 15,1 & 84,9 & $<0,001$ \\
\hline & $\mathrm{RN}$ a termo & 64,3 & 35,7 & & 28,1 & 71,9 & \\
\hline
\end{tabular}

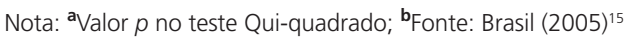

AM: Aleitamento Materno; HAC: Hospital Amigo da Criança; RN: Recém-nascido.

ração aos grandes hospitais da capital (7,3\%) e aos outros hospitais $(12,3 \%) ; p<0,001$ - dados não apresentados em tabela. O parto cesáreo foi menos frequente entre as mães residentes em Belo Horizonte $(57,1 \%)$ e na região metropolitana $(52,6 \%)$, em comparação às residentes nas de- mais cidades do interior do estado $(67,7 \%)$; $p<0,001$ - dados não apresentados em tabela. 0 mesmo foi observado quanto à presença de complicações no parto: Belo Horizonte - 10\%, região metropolitana - $14 \%$ e demais cidades - $24,2 \%$; $p<0,001$ - dados não apresentados em tabela. 
Na análise multivariada final, as variáveis que permaneceram associadas à maior prevalência do "contato pele a pele mãe/filho" foram: modelo 1 - residência materna na região metropolitana e amamentação anterior; modelo 2 - realização de quatro ou mais consultas pré-natais e recebimento de orientação sobre AM no pré-natal (individual + em grupo); modelo 3 - parto normal, ausência de complicações no parto, peso ao nascer adequado e idade gestacional a termo. Já em relação ao local do parto, observou-se menor prevalência do contato mãe/bebê entre as mulheres cujo procedimento foi realizado em grandes hospitais da capital (Tabela 3).
No tocante à amamentação na sala de parto, permaneceram associadas à maior prevalência dessa prática: modelo1 - mães que amamentaram anteriormente ( $\mathrm{RP}=1,22$; Intervalo de Confiança 95\% - IC95\% 1,10-1,35); modelo 2 - as que receberam orientação (individual + em grupo) sobre AM no pré-natal ( $R P=1,26$; IC95\% 1,14-1,40); modelo 3 - mães cujos filhos nasceram de parto normal (RP=1,63; IC95\% 1,45-1,83), as que não tiveram complicações no parto $(R P=1,27$; IC95\% 1,03-1,56), as que tiveram bebê com peso ao nascer adequado ( $\mathrm{RP}=1,92$; IC95\% 1,48-2,48) e nascidos a termo ( $R P=1,40$; IC95\% 1,17-1,67). Ao contrário, notou-se menor prevalência dessa prática entre mulheres que tiveram seus filhos nos

Tabela 3. Razões de prevalências entre as variáveis e o desfecho "contato pele a pele" (modelo final). Banco de Leite Humano, Belo Horizonte (MG), 2009-2012.

\begin{tabular}{|c|c|c|c|c|c|c|}
\hline \multirow{3}{*}{ Modelo } & \multirow{3}{*}{\multicolumn{2}{|c|}{ Variáveis }} & \multicolumn{4}{|c|}{ Passo 4} \\
\hline & & & \multicolumn{2}{|c|}{ Contato pele a pele } & \multicolumn{2}{|c|}{ AM na sala de parto } \\
\hline & & & $\mathrm{RP}$ & IC $95 \%$ & $\mathrm{RP}$ & IC 95\% \\
\hline \multirow{5}{*}{1} & \multirow{3}{*}{ Local de residência } & Belo Horizonte & 1,21 & $1,02-1,44$ & - & - \\
\hline & & Região metropolitana & 1,23 & $1,03-1,46$ & - & - \\
\hline & & Demais cidades & 1,00 & - & - & - \\
\hline & \multirow{2}{*}{ Amamentação anterior } & $\operatorname{sim}$ & 1,07 & $1,02-1,12$ & 1,22 & $1,10-1,35$ \\
\hline & & Não & 1,00 & - & 1 & - \\
\hline \multirow{5}{*}{2} & \multirow{3}{*}{ Número de consultas pré-nataisa } & Até 3 & 1,00 & - & - & - \\
\hline & & 4 a 6 & 1,50 & $1,12-2,02$ & - & - \\
\hline & & Acima de 6 & 1,48 & $1,11-1,98$ & - & - \\
\hline & \multirow{2}{*}{ Orientação sobre AM } & $\operatorname{sim}$ & 1,14 & $1,08-1,20$ & 1,26 & $1,14-1,40$ \\
\hline & & Não & 1,00 & - & 1 & - \\
\hline \multirow{12}{*}{3} & \multirow{3}{*}{ Tipo de parto } & Normal & 1,34 & $1,27-1,41$ & 1,63 & $1,45-1,83$ \\
\hline & & Fórceps & 1,31 & $1,15-1,49$ & 1,03 & $0,71-1,49$ \\
\hline & & Cesárea & 1,00 & - & 1 & - \\
\hline & \multirow{2}{*}{ Complicação no parto } & $\operatorname{sim}$ & 1,00 & - & 1 & - \\
\hline & & Não & 1,24 & $1,12-1,38$ & 1,27 & $1,03-1,56$ \\
\hline & \multirow{3}{*}{ Tipo de hospital } & HAC & 1,02 & $0,95-1,10$ & 0,81 & $0,71-0,92$ \\
\hline & & Grandes hospitais da capital & 0,89 & $0,82-0,97$ & 0,41 & $0,34-0,49$ \\
\hline & & Outros & 1,00 & - & 1 & - \\
\hline & \multirow[b]{2}{*}{ Peso ao nascer } & RN Baixo peso & 1,00 & - & 1 & - \\
\hline & & RN Peso adequado & 1,23 & $1,11-1,36$ & 1,92 & $1,48-2,48$ \\
\hline & \multirow{2}{*}{ Idade gestacional } & Pré-termo & 1,00 & - & 1 & - \\
\hline & & A termo & 1,18 & $1,10-1,28$ & 1,40 & $1,17-1,67$ \\
\hline
\end{tabular}

Nota: a Fonte: Brasil (2005) 15

AM: Aleitamento Materno; HAC: Hospital Amigo da Criança; IC: Intervalo de Confiança; RN: Recém-nascido; RP: Razão de Prevalência. 
grandes hospitais da capital (RP=0,41; IC95\% 0,34-0,49) (Tabela 3). Os resultados da análise multivariada e a integração entre os modelos estão sintetizados na Figura 2.

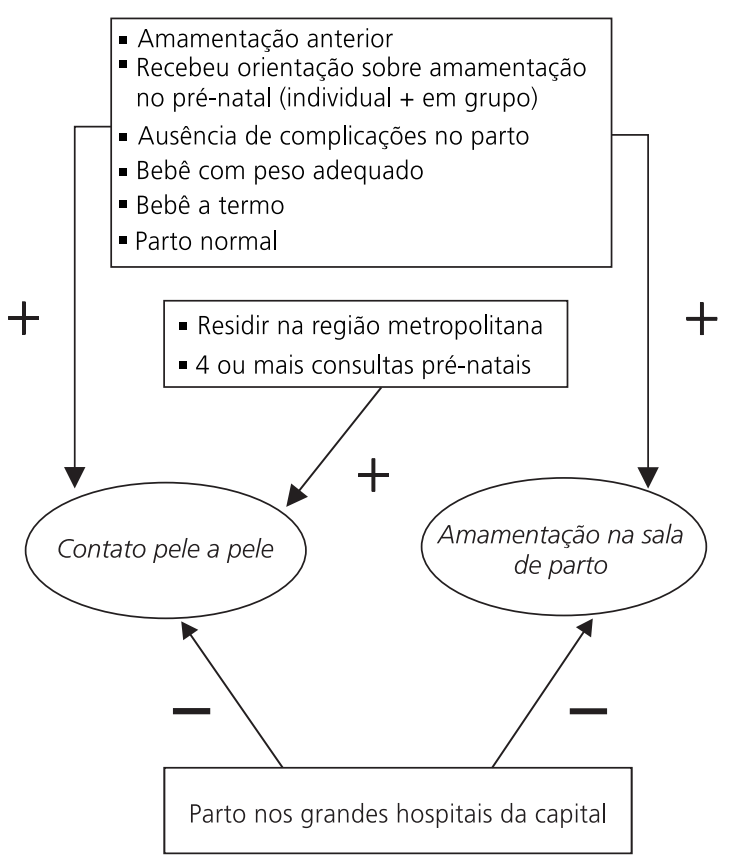

Figura 2. Síntese dos resultados e integração entre os modelos multivariados - associações negativas e positivas com os desfechos. Banco de Leite Humano, Belo Horizonte (MG), 2009-2012.

\section{I S C U S S Ã O}

Neste estudo, constatou-se a presença preponderante dos fatores relacionados à assistência hospitalar e ao recém-nascido, além das orientações sobre AM fornecidas às mães durante o pré-natal e a experiência prévia com amamentação explicando os desfechos. Esses achados reforçam a hipótese da forte influência das práticas institucionais e das condições do bebê ao nascimento tanto no contato pele a pele precoce entre mãe e filho quanto no início da amamentação na sala de parto.

A importância dessas práticas, segundo recomendação da OMS e da Iniciativa Hospital Amigo da Criança², tem sido bem documentada, apesar da baixa frequência na amostra estudada (amamentação na sala de parto - 25,5\%). Esse resultado assemelha-se aos de Moreira et al. ${ }^{11}$, que identificaram, em hospitais com o título de Hospital Amigo da Criança, baixa frequência da amamentação na sala de parto (24\%).

Assim, o fato de mais de quatro consultas pré-natais ter favorecido o contato pele a pele, na presente pesquisa pode estar relacionado às evidências de que o maior número de consultas se associa positivamente ao maior conhecimento e ao sucesso do AM. Em um ambulatório materno-infantil em Tubarão (SC), as gestantes as quais mencionaram terem realizado quatro ou mais consultas pré-natais tiveram uma prevalência $40 \%$ superior de informações sobre amamentação, quando comparadas com aquelas que realizaram menos consultas ${ }^{16}$. Isso aponta o pré-natal como momento oportuno para explicar a importância do contato pele a pele mãe/filho ${ }^{6}$, já que tem como um dos seus objetivos o esclarecimento quanto às novas situações pelas quais essas mulheres e seus familiares irão vivenciar, desde a gestação, até o período após o nascimento do bebê.

Outro fator observado nesta pesquisa e que favoreceu tanto o contato pele a pele quanto a amamentação na sala de parto, foi a experiência prévia em aleitamento materno. Há evidências de que o contato precoce nutriz/bebê e a amamentação anterior estão associados a um alvo comum: a maior duração do AM em curto ou longo prazos ${ }^{5,17}$. As mulheres que já tiveram uma experiência anterior favorável em amamentação se mostram mais propensas ao aleitamento, possivelmente por terem maior facilidade e segurança para realizá-lo com os demais filhos ${ }^{17}$. De modo semelhante, o contato precoce pele a pele pode promover o AM pelo estímulo à produção de ocitocina, hormônio responsável pela ejeção de leite e favorecimento do vínculo mãe/bebê, que é importante para o início e manutenção do aleitamento materno exclusivo ${ }^{5}$.

A experiência prévia em amamentação também representou fator protetor ao aleita- 
mento na sala de parto, possivelmente pela maior valorização do AM por parte das nutrizes. Segundo Barbosa et al. ${ }^{18}$, a vivência da amamentação, associada ao reconhecimento dos valores nutritivos e afetivos advindos da prática, auxiliam na sua continuidade e na valorização da experiência de amamentar logo após o parto.

Corroborando os achados desta pesquisa, os mesmos autores afirmaram que a falta de orientação das nutrizes, por parte dos profissionais de saúde, quanto à amamentação na sala de parto e ao contato mãe-bebê, colabora para a redução dessas práticas e do aleitamento materno exclusivo. Acrescentam, ainda, que é durante o pré-natal ou mesmo na sala de pré-parto que as gestantes devem ser orientadas, a fim de que esses procedimentos sejam realizados rotineiramente em todas as maternidades ${ }^{18}$.

Outro fator identificado neste estudo e que pode contribuir para a promoção do AM na sala de parto e o contato pele a pele é o nascimento por parto normal, possivelmente por ser um procedimento que não oferece barreiras à amamentação na primeira hora de vida, quando comparado à cesariana. Esta tem sido apontada como importante obstáculo para o início do AM antes ou depois da primeira hora, e está geralmente relacionada a rotinas de cuidados pós-operatórios que adiam ou suspendem o contato entre a mãe e o bebê após o nascimento ${ }^{19}$.

Vieira et al. ${ }^{20}$ afirmam que a primeira limitação com relação ao parto por cesárea diz respeito à capacidade da mãe de tocar o recém-nascido, caso os braços sejam restringidos durante a cirurgia. Outro aspecto diz respeito à analgesia, a qual pode causar um comportamento desorganizado no bebê e prejuízo na busca espontânea pelo seio da nutriz após o nascimento. Entretanto, cumpre salientar que o parto cesariano não deve ser visto como uma contraindicação para a amamentação na sala de parto, já que o aleitamento materno proporciona benefícios adicionais para a mãe, como a produção de ocitocina, que reduz o sangramento puerperal e acelera a involução uterina $^{19}$.
Em partos com complicações, as quais favorecem a internação do recém-nascido em unidade de tratamento neonatal, a prática do AM na sala de parto pode ser prejudicada. Esse fato pôde ser observado em uma Unidade Neonatal, na qual as mães enfrentam uma série de obstáculos que podem interferir no início e estabelecimento da amamentação ${ }^{21}$. Estes se relacionavam à ambiência hospitalar, como a falta de conforto e privacidade para amamentar; à assistência profissional, destacando-se a pouca eficácia de orientações e apoio ao AM, particularmente, quando realizadas de forma verticalizada e impositiva; medo e insegurança decorrentes de dificuldades no manejo da amamentação e condição clínica da criança.

A ausência de complicações ao nascimento também pode constituir um fator favorável ao contato pele a pele. Segundo Narchi et al.22, quando ocorre a separação entre mães e filhos após o parto, supõe-se a existência de intercorrências à saúde materna ou neonatal. Porém, os autores ressaltam que mesmo nos casos em que a amamentação é contraindicada, o contato pele a pele deve ser encorajado, não havendo motivos que justifiquem a separação. Em uma comunidade carente de São Paulo, em apenas 8\% dos casos com separação do binômio mãe/filho após o parto a mesma ocorreu devido a complicações que obrigaram os recém-nascidos a permanecerem em berçário por vários dias. Nos demais, não foi encontrada justificativa admissível para a separação.

Nesse contexto, salienta-se que a menor frequência de partos cesáreos e de complicações no parto observada entre as mulheres residentes na capital estadual e região metropolitana pode justificar a maior prevalência do contato pele a pele nutriz/bebê entre as mesmas. A região metropolitana incorpora um conjunto de municípios próximos entre si, os quais são integrados socioeconomicamente a uma cidade central, metrópole, representada, neste estudo, pela cidade de Belo Horizonte ${ }^{23}$.

Da mesma forma, a condição de nascer com o peso adequado ou a termo favorece a 
prática do contato pele a pele e da amamentação na sala de parto, provavelmente devido à dificuldade que os bebês com baixo peso ou prematuros apresentam em relação ao aleitamento materno e à demanda por cuidados especiais da equipe de saúde logo após o parto. Os recém-nascidos com baixo peso ao nascer apresentam maior dificuldade para sugar diretamente o seio materno, além de serem frequentemente separados de suas mães imediatamente após o nascimento e levados para as Unidades de Tratamento Intensivo Neonatais ${ }^{24}$.

Já em relação à idade gestacional, esta pode influenciar as habilidades orais de alimentação e os padrões de sucção em prematuros, podendo, portanto, afetar a progressão da alimentação oral desses bebês ${ }^{25}$. Ademais, lactentes saudáveis, nascidos a termo, apresentam comportamentos inatos que se manifestam imediatamente após o nascimento, quando colocados em contato pele a pele com suas mães. Os recém-nascidos localizam o mamilo através do cheiro, pois apresentam um estímulo intenso ao odor, o qual oferece pistas para iniciar o AM.

Além disso, o fato dos partos realizados nos grandes hospitais da capital terem demonstrado associação negativa com a amamentação na sala de parto e com o contato mãe/filho pode estar relacionado a variações de condutas e normas nas rotinas hospitalares, além do despreparo da equipe de saúde com respeito à realização desses procedimentos. Sabe-se que o excesso de intervenções no período pós-parto pode afetar o início precoce do AM, o qual, dentre outras práticas de assistência ao parto e ao recém-nascido, pode sofrer grande variação entre os serviços de saúde. Estas podem influenciar a qualidade do cuidado ofertado e vêm sendo atribuídas a vários fatores, como a opção médica individual, conhecida como "estilo de prática", desigualdades geográficas e sociais, características demográficas dos pacientes, questões econômicas e culturais, de liderança, entre outras ${ }^{11}$.

Entretanto, em 75\% dos 135 partos realizados em uma sala de Pré-Parto, Parto e Pós-Parto
(PPP) de um hospital público universitário, o bebê foi colocado para mamar ainda no ambiente de parto ${ }^{26}$. Essa sala, de acordo com a RDC 36/2008 da Agência Nacional de Vigilância Sanitária ${ }^{27}$, destina-se a humanizar o atendimento ao parto, resgatando o vínculo mãe/filho, e preconiza, dentre outras ações, o estímulo ao AM no local do parto. No entanto, para isso, são necessárias maior integração e valorização da mulher e do recém-nascido por parte dos profissionais de saúde ${ }^{26}$.

Ressalta-se que o fato do Hospital Amigo da Criança sede do BLH estudado na presente pesquisa ter apresentado maior frequência de complicações no parto em comparação aos outros hospitais pode justificar a baixa prevalência de AM na sala de parto. Essa alta prevalência de complicações pode decorrer do fato da maternidade em questão ser referência para gestações de alto risco, aumentando, consequentemente, a possibilidade de assistência pré-natal a mães com complicações.

Por fim, apesar dos importantes achados, aponta-se as limitações dos estudos de caráter retrospectivo com respeito ao viés de memória e exatidão das informações, neste caso, referentes às mães atendidas no BLH. Nesse sentido, também destaca-se o delineamento do estudo, sujeito à causalidade reversa e ao uso de dados secundários pela ausência de informações em alguns formulários de atendimento. Além disso, pode-se inferir viés quanto ao local do estudo, por ser este detentor do título de Hospital Amigo da Criança e atender mulheres de diversas procedências e contextos. No entanto, essa realidade se assemelha a inúmeras maternidades do país, as quais são alvo da abordagem dos Dez Passos para o Sucesso do Aleitamento Materno. Como potencialidade, ressalta-se o grande número da amostra e a importância do tema no contexto da atenção humanizada ao recém-nascido, destacando os fatores que interferem no contato pele a pele precoce e na amamentação na sala de parto. 


\section{CONCLUSÃ O}

Este estudo sugere a importância do pré-natal como momento oportuno para informar as gestantes sobre o contato pele a pele e a amamentação na sala de parto. Também mostra a relevância da experiência prévia em AM, bem como o fato da gestante residir na região metropolitana como fatores que predispõe o contato pele a pele, provavelmente pelos fatores associados a essas condições.

Ademais, destacam-se o estímulo ao parto normal e a termo e a realização do pré-natal adequado no cumprimento dessas práticas. Entretanto, ressalta-se a necessidade da adoção de medidas as quais priorizem o contato pele a pele e o AM na sala de parto nos grandes hospitais da capital, como a redução ou adiamento de intervenções na assistência pós-parto, além do treinamento e conscientização da equipe de saúde sobre a importância dessas práticas. Estudos longitudinais poderão esclarecer com maior profundidade as questões ainda não elucidadas sobre o assunto, bem como o acompanhamento avaliado da implementação de políticas acerca da temática.

\section{A GRADECIMENTOS}

À Fundação de Amparo à Pesquisa do Estado de Minas Gerais pelo suporte financeiro prestado a este estudo (Processo n APQ-01782-10).

\section{COLABORADORES}

CM SILVA participou da coleta de dados, concepção e delineamento do estudo, análise e interpretação dos dados, elaboração e redação do manuscrito. SCL PEREIRA e IR PASSOS realizaram a revisão crítica do conteúdo intelectual do manuscrito. LC SANTOS participou da concepção e delineamento do estudo, análise e interpretação dos dados, revisão crítica do conteúdo intelectual do manuscrito e aprovação final da versão a ser publicada. Todos os autores leram e aprovaram o manuscrito final.

\section{REFERÊ N CIAS}

1. Boccolini CS, Carvalho ML, Oliveira MIC, Vasconcellos AGG. Fatores associados à amamentação na primeira hora de vida. Rev Saúde Pública. 2011; 45(1):69-78. http://dx.doi.org/10.1590/S0034-89 102010005000051

2. Figueiredo SF, Mattar MJG, Abrão ACFV. Iniciativa Hospital Amigo da Criança - uma política de promoção, proteção e apoio ao aleitamento materno. Acta Paul Enferm. 2012; 25(3):459-63. http://dx. doi.org/10.1590/S0103-21002012000300022

3. Brasil. Ministério da Saúde. Secretaria de Atenção à Saúde. Iniciativa Hospital Amigo da Criança. Brasília; 2011 [acesso 2015 nov 3]. Disponível em: http://bvsms.saude.gov.br/bvs/publicacoes/ iniciativa_hospital_amigo_crianca.pdf

4. Santos LM, Silva JCR, Carvalho ESS, Carneiro AJS, Santana RCB, Fonseca MCC. Vivenciando o contato pele a pele com o recém-nascido no pós-parto como um ato mecânico. Rev Bras Enferm. 2014; 67(2):202-07. http://dx.doi.org/10.5935/0034-71 67.20140026

5. Almeida EA, Filho JM. O contato precoce mãe-filho e sua contribuição para o sucesso do aleitamento materno. Rev Ciênc Méd. 2004; 13(4):381-88.

6. Matos TA, Souza MS, Santos EKA, Velho MB, Seibert ERC, Martins NM. Contato precoce pele a pele entre mãe e filho: significado para mães e contribuições para a enfermagem. Rev Bras Enferm. 2010; 63(6):998-1004. http://dx.doi.org/10.1590/S 0034-71672010000600020

7. Monte GCSB, Leal LP, Pontes CM. Avaliação do $4^{\circ}$ passo para promoção do aleitamento materno em Hospital Amigo da Criança. Rev RENE. 2012; 13(4):861-70.

8. Organização Pan-Americana da Saúde. Organização Mundial da Saúde. Semana Mundial do Aleitamento Materno 2012. Entendendo o passado: planejando o futuro. Comemoração dos 10 anos da Estratégia Global da OMS/UNICEF para alimentação de lactentes e crianças na primeira infância. 2012 [acesso 2015 nov 3]. Disponível em: http://www. paho.org/bra/index.php?option=com_docman\& task $=$ doc_view\&gid $=1445 \&$ ltemid $=423$

9. Brasil. Ministério da Saúde. Pesquisa Nacional de Demografia e Saúde da Criança e da Mulher - PNDS 2006: dimensões do processo reprodutivo e da saúde da criança. Série G. Estatística e Informação em Saúde. Brasília; 2009 [acesso 2015 nov 3]. Disponível em: http://bvsms.saude.gov.br/bvs/ publicacoes/pnds_crianca_mulher.pdf

10. Brasil. Ministério da Saúde. Secretaria de Atenção à Saúde. II Pesquisa de prevalência de aleitamento 
materno nas capitais brasileiras e Distrito Federal. Série C. Projetos, programas e relatórios. Brasília; 2009 [acesso 2015 nov 3]. Disponível em: http:// www.redeblh.fiocruz.br/media/pesquisa.pdf

11. Moreira MEL, Gama SGN, Pereira APE, Silva AAM, Lansky S, Pinheiro RS, et al. Práticas de atenção hospitalar ao recém-nascido saudável no Brasil. Cad Saúde Pública. 2014; 30(Suppl.1):S128-S139. http:// dx.doi.org/10.1590/0102-311X00145213

12. Santos DT, Vannuchi MTO, Oliveira MMB, Dalmas JC. Perfil das doadoras de leite do banco de leite humano de um hospital universitário. Acta Sci, Health Sci. 2009; 31(1):15-21. http://dx.doi.org/10. 4025/actascihealthsci.v31i1.891

13. Fundação Hospitalar do Estado de Minas Gerais. Maternidade Odete Valadares: histórico. [acesso 2016 fev 29]. Disponível em: http://www. fhemig. mg.gov.br/pt/atendimento-hospitalar/complexode-especialidades/maternidade-odete-valadares

14. Fundação Hospitalar do Estado de Minas Gerais. Banco de leite humano: Quem somos. 2012 [acesso 2015 nov 3]. Disponível em: http://www.fhemig. mg.gov.br/pt/banco-de-leite-humano/quem-somos

15. Brasil. Ministério da Saúde. Secretaria de Atenção à Saúde. Pré-natal e puerpério: atenção qualificada e humanizada. Série A. Direitos sexuais e direitos reprodutivos. Caderno n 5. Brasília; 2005 [acesso 2015 nov 3]. Disponível em: http://bvsms.saude. gov.br/bvs/publicacoes/pre-natal_puerperio_ atencao_humanizada.pdf

16. Volpato SE, Braun A, Pegorim RM, Ferreira DC, Beduschi CS, Souza KM. Avaliação do conhecimento da mãe em relação ao aleitamento materno durante o período pré-natal em gestantes atendidas no Ambulatório Materno infantil em Tubarão (SC). ACM Arq Catarin Med. 2009; 38(1):49-55.

17. Faleiros FTV, Trezza EMC, Carandina L. Aleitamento materno: fatores de influência na sua decisão e duração. Rev Nutr. 2006; 19(5):623-30. http://dx. doi.org/10.1590/S1415-52732006000500010

18. Barbosa V, Orlandi FS, Dupas G, Beretta MIR, Fabbro MRC. Aleitamento na sala de parto: a vivência da puérpera. Ciênc Cuid Saúde. 2010; 9(2):366-73. http://dx.doi.org/10.4025/cienccuidsaude. v9i2.11249

19. Coutinho ACFP, Soares ACO, Fernandes PS. Knowledge of mothers about the benefits of breastfeeding to women's health. J Nurs UFPE. 2014; 8(5):1213-20. http://dx.doi.org/10.5205/ reuol.5863-50531-1-ED.0805201415
20. Vieira TO, Vieira GO, Giugliani ERJ, Mendes CMC, Martins CC, Silva L.R. Determinants of breastfeeding initiation within the first hour of life in a Brazilian population: cross-sectional study. BMC Public Health. 2010; 10:760. http://dx.doi.org/10.1186/ 1471-2458-10-760

21. Souza KV, Tesin RR, Alves VH. Mães de recém-nascidos hospitalizados: em/entre círculos no processo de amamentação. Acta Paul Enferm. 2010; 23(5):608-13. http://dx.doi.org/10.1590/s0103-2 1002010000500004

22. Narchi NZ, Fernandes RAQ, Dias LA, Novais DH. Variáveis que influenciam a manutenção do aleitamento materno exclusivo. Rev Esc Enferm USP. 2009; 43(1):87-94. http://dx.doi.org/10.1590/S 0080-62342009000100011

23. Prefeitura de Belo Horizonte. Região metropolitana de Belo Horizonte. 2016 [acesso 2016 mar 4]. Disponível em: http://gestao compartilhada.pbh.gov. br/estrutura-territorial/regiao-metropolitana-debelo-horizonte

24. Mamemoto K, Kubota M, Nagai A, Takahashi Y, Kamamoto T, Minowa $\mathrm{H}$, et al. Factors associated with exclusive breastfeeding in low birth weight infants at NICU discharge and the start of complementary feeding. Asia Pac J Clin Nutr. 2013; 22(2):270-75. http://dx.doi.org/10.6133/apjcn.20 13.22.2.11

25. White-Traut R, Pham T, Yoder J. Exploring factors related to oral feeding progression in premature infants. Adv Neonatal Care. 2013; 13(4):288-94. http://dx.doi.org/10.1097/ANC.0b013e3182 $9 d 8 c 5 a$

26. Stancato K, Vergílio MSTG, Bosco CS. Avaliação da estrutura e assistência em sala de pré-parto, parto e pós-parto imediato - PPP de um hospital universitário. Ciênc Cuid Saúde. 2011; 10(3):541-48. http://dx.doi.org/10.4025/cienccuidsaude. v10i3.12656

27. Brasil. Ministério da Saúde. Agência Nacional de Vigilância Sanitária. Resolução $n^{\circ} 36$, de 3 de junho de 2008. Dispõe sobre regulamento técnico para funcionamento dos serviços de atenção obstétrica e neonatal. Brasília; 2008 [acesso 2015 nov 3]. Disponível em: http://bvsms.saude.gov.br/bvs/ saudelegis/anvisa/2008/res0036_03_06_2008_ rep.html

Recebido: novembro 12, 2015 Versão final: março 23, 2016 Aprovado: maio 3, 2016 
